\title{
Ultrasound evaluation of mediastinal lymphadenopathy in patients with sarcoidosis
}

\author{
Tim O. Hirche ${ }^{1}$, Herbert Hirche ${ }^{2}$, Xin-Wu Cui ${ }^{3}$, Thomas O. Wagner ${ }^{4}$, Christoph F. Dietrich ${ }^{3}$
}

${ }^{1}$ Department of Pulmonary Medicine, German Clinics for Diagnostics (DKD), Wiesbaden, ${ }^{2}$ Institute for Medical Informatics, Biometry and Epidemiology, University of Duisburg-Essen ${ }^{3}$ Department of Internal Medicine II, Caritas Hospital, Bad Mergentheim, ${ }^{4}$ Department of Pulmonary Medicine, J. W. Goethe University, Frankfurt am Main, Germany

\begin{abstract}
Aim: Mediastinal lymphadenopathy is a typical feature of pulmonary sarcoidosis and an important parameter for diagnosis and follow-up. The present feasibility study is the first to elucidate the role of transthoracic mediastinal ultrasonography (US) for evaluation and staging of lymphadenopathy in patients with sarcoidosis. Material and method: Fifty patients with sarcoidosis were subjected to high-definition mediastinal US. The sonographic lymph node status was compared with the radiologic staging - the prevailing gold standard. Results: Mediastinal regions and landmarks could reliably be assessed by ultrasound in 45/50 (90\%) of sarcoidosis patients. Lymphadenopathy was sonographically documented in 29/50 (58\%) of the patients (sensitivity $89 \%$, specificity $76 \%$, PPV 86\%, NPV 81\%, accuracy $84 \%$ ). There was a marked concordance between US confirmation of lymphadenopathy and radiologic staging $(\kappa=0.67, p<0.001)$. Conclusions: Transthoracic US qualifies for the demonstration of the mediastinal regions and lymphadenopathy in patients with sarcoidosis. The procedure is facilitated by frequent and distinct mediastinal lymph node enlargement due to sarcoidosis. Prospective studies are required to find out whether mediastinal US adds value to conventional radiologic staging and provides a clinically advantage, particularly in the follow-up of patients with sarcoidosis.
\end{abstract}

Keywords: ultrasonography, mediastinum, chest, sarcoidosis, lymphadenopathy.

\section{Introduction}

Sarcoidosis (Boeck's disease) is a systemic soft tissue disorder with formation of granulomas. Its etiology has thus far not clearly been elucidated [1]. Infectious as well as autoimmune processes are being discussed as causative factors. Aside from acute manifestations and flaires, many patients experience a gradual chronic course with

Received 03.05.2014 Accepted 28.05.2014

Med Ultrason

2014, Vol. 16, No 3, 194-200

Corresponding author: Prof. Dr. med. Christoph F. Dietrich Department of Internal Medicine II, Caritas Hospital

Uhland Str. 7

97980 Bad Mergentheim /Germany

Phone: +497931582201

E-mail: christoph.dietrich@ckbm.de only a few symptoms. Sarcoidosis may principally affect any organ system, but in $90 \%$ of the cases thoracic lymph nodes and/or the lungs are involved. Computerized tomography of the chest is nowadays considered the gold standard for diagnosis of mediastinal and pulmonary alterations [2]. This method, however, is flawed by a high radiation exposure, expenses and limited availability. Apart from clinical and laboratory parameters (e.g. angiotensin-converting enzyme, soluble IL-2 receptor), conventional chest X-ray is the most commonly used tool, particularly for follow-up of sarcoidosis patients, although the imaging resolution is often insufficient to evaluate mediastinal structures $[3,4]$.

Based on radiologic findings, sarcoidosis of the lungs is classified in stages I-IV, with a differentiation between mediastinal lymphadenopathy with and without involvement of the pulmonary parenchyma. The attribution to 
stages is an epidemiologic factor; though its clinical and prognostic relevance for the individual patient is rather limited $[5,6]$.

In recent years many studies have explored the feasibility of endoscopic ultrasonography (EUS/EBUS) for diagnosis of sarcoidosis. Aside from improved mediastinal staging, these methods are especially suited for the cyto-/histopathologic verification of the underlying disease (e.g., EBUS-TBNA) [7-13]. However, in view of relative expenditure and invasiveness, these procedures are not practicable in the follow-up of sarcoidosis patients.

Transthoracic US has still been rarely used in clinical routine, despite the fact that modern high-definition US devices and facultative employment of color duplex US permit an accurate discrimination of mediastinal regions and cardinal structures [14]. Initial studies, comparing the feasibility and accuracy of suprasternal and parasternal mediastinal ultrasound examination with the results of computer-assisted tomography, proved sensitivities between 69 and $100 \%$ in the demonstration of pathologically enlarged mediastinal lymph nodes $[15,16]$. Mediastinal US has since been validated and applied in model $[17,18]$, post mortem examinations [19], healthy subjects [19], children [20], patients with cystic fibrosis [21], hepatitis C [22] and in the follow-up on malignant diseases [23,24].

Transthoracic mediastinal US has to date not been studied in sarcoidosis patients, although by comparison with other inflammatory disorders, sarcoidosis is particularly characterized by prominent enlargement of the mediastinal lymph nodes. The present study focuses on the US demonstration of mediastinal lymph node regions in patients with sarcoidosis and relates the lymph node status obtained on mediastinal US to the radiologic stage.

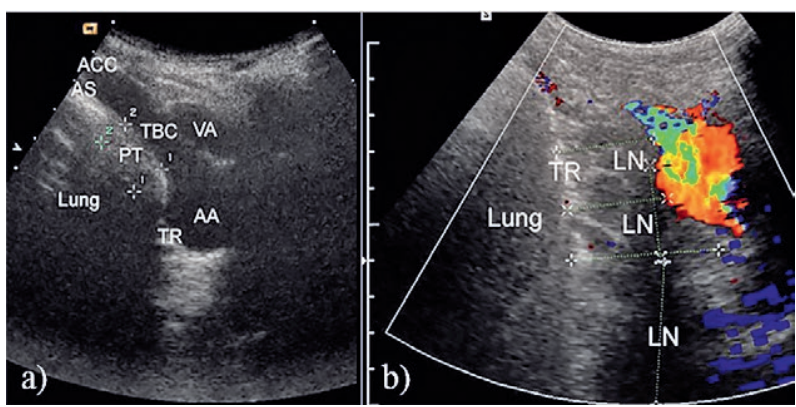

Fig 1. Paratracheal region. Demonstration of the sonographic anatomy in (a) a healthy subject and (b) a patient with sarcoidosis with enlarged mediastinal lymph nodes (LN). The lung, trachea (TR), common carotid artery (ACC), subclavian artery (AS), ascending aorta (AA), brachiocephalic trunk (TBC) and anonymous vein (VA) are indicated.

\section{Patients and methods}

Fifty patients with pulmonary sarcoidosis followed up in our outpatient unit were consecutively enrolled in this study (18 males, 32 females, mean age $\pm \mathrm{SD}, 39$ \pm 9.1 years). The diagnosis of sarcoidosis had been established according to guidelines by clinical, serologic, radiologic and histopathologic findings. In the course of disease the radiologic staging of sarcoidosis was defined by conventional chest X-ray according to Scadding's modified staging system [25], defining stage I as solely hilar and mediastinal lymphadenopathy, stage II as lymphadenopathy and pulmonary infiltration, stage III as exclusively pulmonary infiltration and stage IV as manifest pulmonary fibrosis. Patients with sarcoidosis and clinical evidence of acute exacerbation, airways infection or neoplasia were not included in this study. Written informed consent was obtained from all subjects. The study was conducted according to the ethical standards of our institution and the Declaration of Helsinki and its revisions.

\section{US systems}

The mediastinum of all patients was assessed by high-definition US (3.5-5 MHz transducers, Acuson, Erlangen, Germany). To discriminate atypical vessels from lymph nodes, an additional $7 \mathrm{MHz}$ transducer and/ or color duplex sonography (Acuson or Siemens Elegra, Erlangen, Germany) was applied. All US examinations were carried out by one expert in thoracic and mediastinal sonography and documented.

\section{Method to study the mediastinum}

Mediastinal compartments or regions can be sonographically differentiated by cardinal structures, vessels and lymph node stations (fig 1, fig 2). US examination of these regions followed specifically validated and pub-

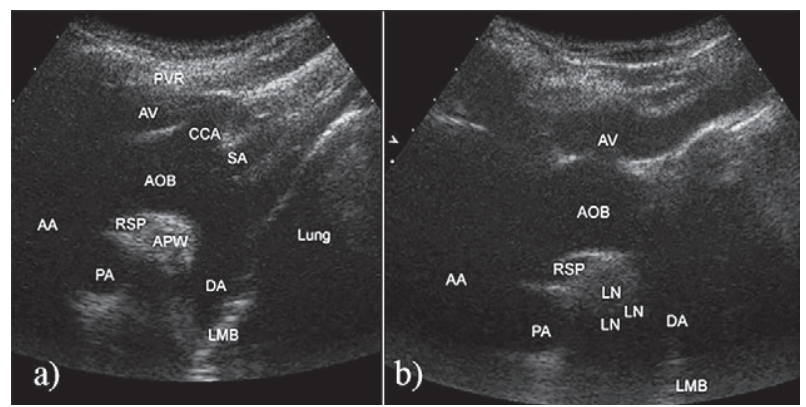

Fig 2. Aortopulmonary window. Demonstration of the sonographic anatomy in (a) a healthy subject and (b) a patient with sarcoidosis with enlarged mediastinal lymph nodes (LN). The ascending aorta (AA), aortic bow (arch, AOB) descending aorta (DA), pulmonary artery (PA), common carotid artery (CCA), subclavian artery (SA), anonymous vein (AV), recessus superior pericardii (RSP), left main bronchus (LMB), prevascular region (PVR), and lung are indicated. 
Table I. Criteria for adequate sonomorphologic evaluation of the mediastinal regions.

\begin{tabular}{ll}
\hline Region & Criteria of assessability \\
\hline Supraaortic region & $\begin{array}{l}\text { Suprasternal presentation of the complete aorta arch with all major supraaortic branches, presentation } \\
\text { of the anonymous vein and both brachiocephalic veins with outlets into the superior caval vein }\end{array}$ \\
Paratracheal region & $\begin{array}{l}\text { Suprasternal presentation of the brachiocephalic trunk, the brachiocephalic vein, ascending aorta and } \\
\text { right pulmonary artery }\end{array}$ \\
$\begin{array}{l}\text { Aortopulmonary } \\
\text { window }\end{array}$ & $\begin{array}{l}\text { Suprasternal presentation of the entire aortic arch and the pulmonary artery trunk } \\
\text { Prevascular region }\end{array}$ \\
Subcarinal region & $\begin{array}{l}\text { Right and left parasternal presentation of the ascending aorta and pulmonary artery } \\
\text { Right and left parasternal presentation of the ascending aorta, of the right pulmonary artery and left } \\
\text { atrium in 2 planes (in strict lateroposition). The subcarinal region is frequently better visualized on } \\
\text { exhalation and demarcated at the atrial roof } \\
\text { Right and left parasternal presentation of right atrium, right and left ventricle and of the bilateral } \\
\text { adjacent pericardial fatty tissue }\end{array}$ \\
\hline Pericardial region &
\end{tabular}

lished protocols [26]. Briefly, suprasternal mediastinal exploration of the supine patient is carried out by slightly elevating the upper part of the body and by retroverting the head. The transducer is placed right above the sternal manubrium in the jugular fossa. The parasternal region must be accessed with the patient strictly being in either right or left lateral position since this position alone will permit an adequate sonographic approach by mediastinal shifting. The following areas were evaluated: the supraaortic region by the suprasternal semisagittal approach, the paratracheal region by the right suprasternal coronary approach, and the aortopulmonary window by suprasternal semisagittal approach - each with maximum reclination of the head. The prevascular region was assessed by suprasternal and ventral parasternal approach, the subcarinal and pericardial region by bilateral parasternal approach in a strictly lateral position. The posterior mediastinum was not included due to its considerably lesser US accessibility. Particular criteria that were obligatory to ensure proper assessment of the different regions are listed in Table I. When these criteria were not met, mediastinal US was classified unfeasible. Lymph nodes that could be demonstrated on US were measured in their longest vertical axis. In agreement with the published and validated recommendations by anatomic studies, lymph nodes were rated pathologic when their sonographically determined size was $\geq 25 \mathrm{~mm}$ in the longest axis $[19,22]$. What seems to be a discrepancy regarding the liminal value for pathologic mediastinal lymph node size defined by computed tomography $(\geq 10-15 \mathrm{~mm})$ is explained by the anatomic longitudinal arrangement of mediastinal lymph nodes. Contrary to the US approach, radiologic sectional procedures, such as computed tomography, visualize and gauge mediastinal lymph nodes in their transversal short axis [27].

\section{Statistics}

Clinical and biochemical parameters were presented as mean ( \pm standard deviation, $S D)$ or median $(25-75 \%$ percentiles), where appropriate. The statistical criteria of quality sensitivity (SE), specificity (SP), positive predictive value (PPV), negative predictive value (NPV) and accuracy (A) for the US demonstration of mediastinal lymph nodes were computed under the assumption of radiologic findings being the prevailing gold standard for follow-up assessment of mediastinal lymphadenopathy in sarcoidosis patients. The conformity of the results by these two diagnostic procedures was rated according to Cohen's kappa coefficient [28].

Categorical variables were compared by Fisher's exact test, Cochran's trend test and ranking analysis of variance according to Kruskal-Wallis. Correlations between the radiologic and US findings were determined by Spearman's rank-correlation coefficient [29]. To identify parameters associated with US evidence of mediastinal lymph nodes, a model of multiple logistic regression was used by a stepwise forward selection of the variables [30]. Clinical variables (age, gender, height, weight and body mass index [BMI]), along with the time elapsed since first diagnosis, served as independent predictors in the model. Statistical significance was predefined at the level of $\mathrm{a}=0.05$. Empirically found $p$-values are given descriptively.

\section{Results}

Among the 50 sarcoidosis patients participating in this study, $10(20 \%)$ were categorized radiologic stage I, $18(36 \%)$ stage II, $14(28 \%)$ stage III, and $8(16 \%)$ stage IV. US exploration of the mediastinal regions was 


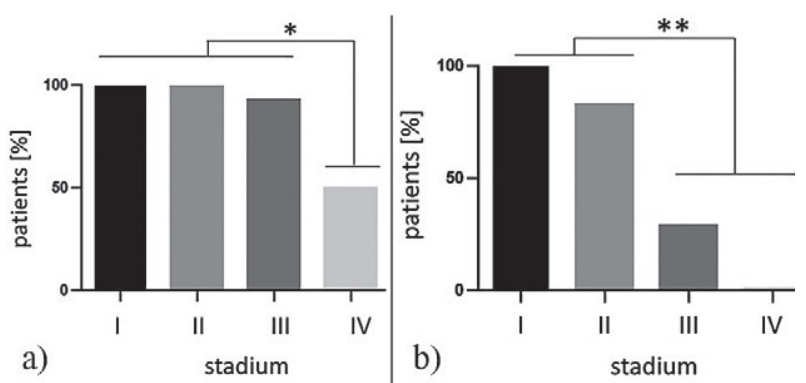

Fig 3. (a) Sonographic presentation of the mediastinal regions $\left({ }^{*} \mathrm{p}<0.002\right)$. b) Evidence of mediastinal lymphadenopathy depending on the stage of sarcoidosis $(* * \mathrm{p}<0.001)$.

Table II. Sonographic detection of mediastinal lymphadenopathy depending on the radiologic stage of pulmonary sarcoidosis.

\begin{tabular}{lccc}
\hline $\begin{array}{l}\text { Sarcoidosis } \\
\text { of the lung } \\
\text { (radiologic } \\
\text { classification) }\end{array}$ & $\begin{array}{c}\text { Total } \\
\text { number of } \\
\text { patients }\end{array}$ & $\begin{array}{c}\text { Sonographic } \\
\text { accessibility } \\
\text { of mediasti- } \\
\text { num }\end{array}$ & $\begin{array}{c}\text { Sonographic } \\
\text { detection } \\
\text { of enlarged } \\
\text { lymph nodes }\end{array}$ \\
\hline All & $50(100 \%)$ & $45 / 50(90 \%)$ & $29 / 50(58 \%)$ \\
Stage I & $10(20 \%)$ & $10 / 10(100 \%)$ & $10 / 10(100 \%)$ \\
Stage II & $18(36 \%)$ & $18 / 18(100 \%)$ & $15 / 18(83 \%)$ \\
Stage III & $14(28 \%)$ & $13 / 14(93 \%)$ & $4 / 14(29 \%)$ \\
Stage IV & $8(16 \%)$ & $4 / 8(50 \%)$ & $0 / 8(0 \%)$ \\
\hline
\end{tabular}

successful in $90 \%$ of the patients with sarcoidosis. The mediastinal regions of patients in the radiologic stages I, II and III were particularly well approached by transthoracic US - contrary to stage IV in whom the mediastinum was not satisfactorily presented by US in the moiety of the patients (table II, fig 3a).

The radiologic finding of mediastinal lymphadenopathy was sonographically confirmed for all 10 patients in stage I. In stage II, the radiologic and US picture was congruent in $15 / 18$ of the cases; in the remaining 3 patients the mediastinal lymph nodes were rated as sonographically unremarkable. In 10/14 of the stage III cases, the radiologic and US finding of unremarkable mediastinal lymph nodes was congruent. In the remaining 4 patients, however, despite unremarkable radiology, mediastinal lymphadenopathia was detected by US examination. In accordance with radiologic results, none of the stage IV patients presented with sonographically enlarged lymph nodes. As expected, US evidence of mediastinal lymphadenopathy was significantly reduced with advancing radiologic stages of sarcoidosis (fig $3 b$ ).

With reference to the radiologic method, mediastinal US diagnosed marginally more pathologically enlarged lymph nodes $(28 / 50$ [56\%] vs. $29 / 50$ [58\%], group dif-

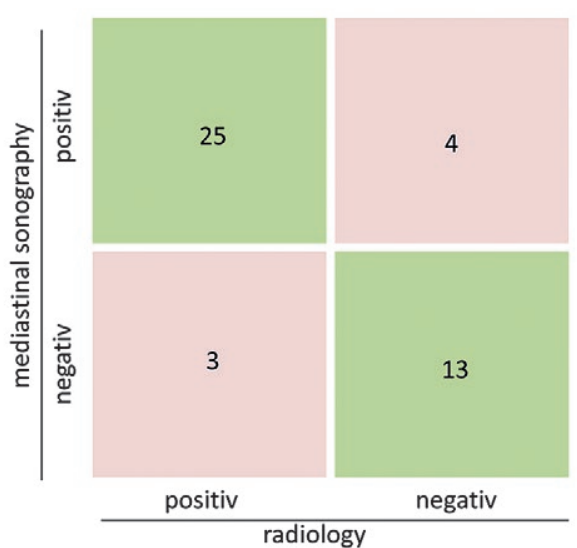

Fig 4. Comparison between the radiologic and sonographic prediction of lymphadenopathy. There is substantial concordance of the two methods in the study group of patients with pulmonary sarcoidosis $(\mathrm{p}<0.001)$.

Table III. Statistic criteria of quality regarding the sonographic detection of mediastinal lymphadenopathy depending on the radiologic stage of sarcoidosis.

\begin{tabular}{lcc}
\hline Mediastinal sonography & No. of patients & \% (95\% CI) \\
\hline Sensitivity & $25 / 28$ & $89.3(72-98)$ \\
Specificity & $13 / 17$ & $76.5(50-93$ \\
Positive predictive value & $25 / 29$ & $86.2(68-96)$ \\
Negative predictive value & $13 / 16$ & $81.3(54-96)$ \\
Accuracy & $(13+25) / 45^{*}$ & $84.4(70-94)$ \\
\hline
\end{tabular}

* $n=45$ patients with sonographically evaluable mediastinal regions

ference n.s.). Compared to the prevailing gold standard of radiologically defined stages, the statistic criteria of quality suggest that US could serve as an equivalent diagnostic parameter to identify mediastinal lymphadenopathy in sarcoidosis (table III). There is an altogether significant agreement of the two diagnostic approaches, with a high index for concordance ( $\mathrm{k}=0.67$, Cohen's coefficient) (fig 4).

There were no significant correlations between the clinical parameters age, gender, height, weight, BMI, as well as time after first diagnosis compared to the radiologic and US staging ( $>0.05$, Spearman's rank-correlation coefficient). Descriptively, when all parameters were entered in a model of multiple logistic regression, the number of sonographically evidenced mediastinal lymph node enlargements decreased with advancing age $(p<0.03)$ and add-on weight $(p<0.07)$; the other clinical parameters were not predictive in the model (data without illustration). 


\section{Discussions}

The present feasibility study was the first attempt to demonstrate that transthoracic mediastinal US examination -validly established in the exploration of other inflammatory diseases - also has a potential in evaluating mediastinal lymphadenopathy in patients with sarcoidosis.

Mediastinal lymph node enlargement is a typical feature of pulmonary sarcoidosis and an important symptom for diagnosis and follow-up. The radiological supported classification of sarcoidosis (stages I-IV) is essentially based on confirmation/exclusion of mediastinal lymphadenopathy [25]. Conventional chest X-ray continues to be the standard diagnostic imaging especially in the follow-up on sarcoidosis patients. However, owing to restricted image resolution and overlying artifacts, the differentiation of mediastinal structures by chest X-ray is limited. A recently published multicenter study involving patients with sarcoidosis revealed a high (interobserver) variability of findings even among skilled radiologists [31].

The radiologic stage of sarcoidosis statistically correlates with the stage of lung disease, and it was postulated that the prognosis worsened with higher score values $[32,33]$. Considering the substantial variation for individual patients, however, one cannot derive statements regarding the future clinical course or suggestions of treatments. A prospective follow-up on 36 patients with sarcoidosis proved that clinically relevant exacerbations of sarcoidosis did not result in significant changes of the radiologic stage [34]. Furthermore, in sarcoidosis patients the conventional radiograph does insufficiently correlate with clinical parameters such as dyspnea [35] or 6 minutes walk test [36].

The weak point of conventional chest X-ray, namely the differentiation of mediastinal structures, might be compensated for by mediastinal US. The latter qualifies well in the follow-up on sarcoidosis patients by the absence of radiation hazards, low costs and broad availability, and it can also be applied complementary to standard radiologic diagnostics. The results of the present study show that in the majority of patients with sarcoidosis mediastinal regions and landmark structures are accessible to transthoracic sonography (90\%). The US presentation in these patients was comparable with the published data of healthy controls (85\%) and other inflammatory diseases with associated lymphadenopathy $(89-93 \%)$ [21,22]. The sonomorphologic evaluation of mediastinal regions was restricted only in stage IV sarcoidosis. In our experience, this phenomenon is essentially due to progressive destruction and scarring of the pulmonary parenchyma and concomitant hyperinflation commonly seen in this stage.

The high sensitivity of US examination for mediastinal lymphadenopathy in the present study (89\%) is likely explained by more pronounced lymph node enlargement in sarcoidosis than in comparable inflammatory diseases [22]. Although US can only identify a fraction of the approximately 60 physiologic mediastinal lymph nodes, pathologically enlarged lymph nodes are typically and synchronously present in several mediastinal regions $[2,37]$. In a recent study with untreated sarcoidosis patients, eight involved intrathoraic lymph node stations were exposed by computed tomography on average. The paratracheal lymph node stations, which are particularly qualified for US demonstration, are pathologically enlarged in up to $80 \%$ of sarcoidosis patients [27].

The results of our study demonstrate a significant concordance between the radiologic and sonomorphologic findings of enlarged mediastinal lymph nodes in sarcoidosis patients. The radiologic and mediastinal US findings in stages I and IV are entirely compatible. Minor deviations of the two methods were found in stages II and III.

Since the radiologic classification of sarcoidosis was defined as the gold standard in this study, deviating sonographic findings were statistically rated as either false negative or false positive. We should note though that in an earlier post mortem study on 20 cadavers, all lymphomas detected on mediastinal US were confirmed by the gold standard of autopsy (specificity 100\%), with anatomically correct determination of lymph nodes in terms of location, shape and size [19]. It can therefore be postulated that the "false positive" US findings of enlarged mediastinal lymph nodes in stage III sarcoidosis are, in fact, consistent with false negative finding of the radiologic gold standard and thus attributable to stage II. This hypothesis points to a potential of mediastinal US - namely, as a complementary procedure to radiologic diagnosis - to ensure a more precise staging in sarcoidosis.

Some limitations of our preliminary study result from a limited and inhomogeneous study population. Future studies should be sufficiently powered to allow for subgroup analysis, e.g. to control for effects of previous treatments versus therapy-naïve patients. CT-scans of the thorax could not be performed in all study subjects due to their objection to high radiation exposure (e.g., in patients where clinical diagnosis is unquestionable, with minor complaints and/or of young age). Subsequent studies should therefore consider alternative imaging technologies as gold standard (e.g. ultra-low-dose CT or MRI scan). Mediastinal US is a highly operator-dependent procedure and simultaneous image acquisition and interpretation requires longstanding 
expertise. Nowadays, US of the chest, and in particular of the mediastinum, is not routinely performed in most centres. Thus, the practical relevance of our findings for routine clinical settings might be questioned. However, despite its constraints, we believe that our data can serve as proof of principle and might help to spark interest in chest sonography in other investigators.

We conclude that mediastinal US is eligible for investigation of lymphadenopathy in patients with sarcoidosis, at least when performed by a skilled investigator using high-quality equipment. Whether mediastinal US adds value to conventional radiologic staging and constitutes a true clinical and prognostic benefit for sarcoidosis patients remains to be evaluated by future studies.

Acknowledgement: Xin-Wu Cui was supported by the Bad Mergentheimer Leberverein e.V.

\section{Conflict of interest: none}

\section{References}

1. Hunninghake GW, Costabel U, Ando M, et al. ATS/ERS/ WASOG statement on sarcoidosis. American Thoracic Society/European Respiratory Society/World Association of Sarcoidosis and other Granulomatous Disorders. Sarcoidosis Vasc Diffuse Lung Dis 1999; 16: 149-173.

2. Sider L, Horton ES Jr. Hilar and mediastinal adenopathy in sarcoidosis as detected by computed tomography. J Thorac Imaging 1990; 5: 77-80.

3. Judson MA, Gilbert GE, Rodgers JK, Greer CF, Schabel SI. The utility of the chest radiograph in diagnosing exacerbations of pulmonary sarcoidosis. Respirology 2008; 13 : 97-102.

4. Judson MA, Thompson BW, Rabin DL, et al. The diagnostic pathway to sarcoidosis. Chest 2003; 123: 406-412.

5. Baughman RP, Culver DA, Judson MA. A concise review of pulmonary sarcoidosis. Am J Respir Crit Care Med 2011; 183: 573-581.

6. Scadding JG. Calcification in sarcoidosis. Tubercle 1961; 42: 121-135.

7. Low A, Medford AR. Endobronchial ultrasound-guided transbronchial needle aspiration. Rev Recent Clin Trials 2013; 8: 61-71.

8. Imai N, Imaizumi K, Ando M, et al. Echoic features of lymph nodes with sarcoidosis determined by endobronchial ultrasound. Intern.Med 2013; 52: 1473-1478.

9. Plit M, Pearson R, Havryk A, Da Costa J, Chang C, Glanville AR. Diagnostic utility of endobronchial ultrasoundguided transbronchial needle aspiration compared with transbronchial and endobronchial biopsy for suspected sarcoidosis. Intern Med J 2012; 42: 434-438.

10. Oki M, Saka H, Kitagawa C, et al. Prospective study of endobronchial ultrasound-guided transbronchial needle as- piration of lymph nodes versus transbronchial lung biopsy of lung tissue for diagnosis of sarcoidosis. J Thorac Cardiovasc Surg 2012; 143: 1324-1329.

11. Navani N, Booth HL, Kocjan G, et al. Combination of endobronchial ultrasound-guided transbronchial needle aspiration with standard bronchoscopic techniques for the diagnosis of stage I and stage II pulmonary sarcoidosis. Respirology 2011; 16: 467-472.

12. Garwood S, Judson MA, Silvestri G, Hoda R, Fraig M, Doelken P. Endobronchial ultrasound for the diagnosis of pulmonary sarcoidosis. Chest 2007; 132: 1298-1304.

13. Dietrich CF. Endobronchialer Ultraschall (EBUS). In: Dietrich CF, Nurnberg D. Interventionelle Sonographie. Thieme Verlag, 2011: 387-399.

14. Dietrich CF, Cui XW, Ignee A, Schreiber-Dietrich D, Hocke M, Hirche TO. Ultrasound of the pleura and lungs. J Ultrasound Med 2014; in press.

15. Genereux GP, Howie JL. Normal mediastinal lymph node size and number: CT and anatomic study. AJR Am J Roentgenol 1984; 142: 1095-1100.

16. Glazer GM, Gross BH, Quint LE, Francis IR, Bookstein FL, Orringer MB. Normal mediastinal lymph nodes: number and size according to American Thoracic Society mapping. AJR Am J Roentgenol 1985; 144: 261-265.

17. Wernecke K, Peters PE, Galanski M. Mediastinal tumors: evaluation with suprasternal sonography. Radiology 1986; 159: 405-409.

18. Wernecke K, Potter R, Peters PE, Koch P. Parasternal mediastinal sonography: sensitivity in the detection of anterior mediastinal and subcarinal tumors. AJR Am J Roentgenol 1988; 150: 1021-1026.

19. Dietrich CF, Liesen M, Buhl R, et al. Detection of normal mediastinal lymph nodes by ultrasonography. Acta Radiol 1997; 38: 965-969.

20. von Lengerke HJ, Schmidt H. Mediastinal sonography in childhood. Results of 310 studies. Radiologe 1988; 28: 460-465.

21. Dietrich CF, Chichakli M, Bargon J, et al. Mediastinal lymph nodes demonstrated by mediastinal sonography: activity marker in patients with cystic fibrosis. J Clin.Ultrasound 1999; 27: 9-14.

22. Dietrich CF, Lee JH, Herrmann G, et al. Enlargement of perihepatic lymph nodes in relation to liver histology and viremia in patients with chronic hepatitis C. Hepatology 1997; 26: 467-472.

23. Wernecke K, Vassallo P, Hoffmann G, et al. Value of sonography in monitoring the therapeutic response of mediastinal lymphoma: comparison with chest radiography and CT. AJR Am J Roentgenol 1991; 156: 265-272.

24. Wernecke K, Diederich S. Sonographic features of mediastinal tumors. AJR Am.J.Roentgenol. 1994; 163: 1357-1364.

25. Scadding JG. Prognosis of intrathoracic sarcoidosis in England. A review of 136 cases after five years' observation. Br Med J 1961; 2: 1165-1172.

26. Dietrich CF, Liesen M, Wehrmann T, Kirchner J, Caspary WF. Mediastinalsonographie, eine neue Bewertung der Befunde. Endoskopie heute 1995; 4: 278-294. 
27. Trisolini R, Anevlavis S, Tinelli C, Orlandi P, Patelli M. CT pattern of lymphadenopathy in untreated patients undergoing bronchoscopy for suspected sarcoidosis. Respir Med 2013; 107: 897-903.

28. Rosner B. Fundamentals of Biostatistics. Duxbury Brooks/ Cole, Pacific Grove, CA USA, 2010.

29. Sachs L. Angewandte Statistik. Springer Verlag; Berlin, Heidelberg, New York, 2003.

30. Statistical Analysis System (SAS Vers 9.1). SAS Institute Inc.; Cary, NC, USA, 2008.

31. Baughman RP, Shipley R, Desai S, et al. Changes in chest roentgenogram of sarcoidosis patients during a clinical trial of infliximab therapy: comparison of different methods of evaluation. Chest 2009; 136: 526-535.

32. Judson MA, Baughman RP, Thompson BW, et al. Two year prognosis of sarcoidosis: the ACCESS experience. SarcoidosisVasc Diffuse Lung Dis 2003; 20: 204-211.
33. Nagai S, Shigematsu M, Hamada K, Izumi T. Clinical courses and prognoses of pulmonary sarcoidosis. Curr Opin Pulm Med 1999; 5: 293-298.

34. Judson MA, Gilbert GE, Rodgers JK, Greer CF, Schabel SI. The utility of the chest radiograph in diagnosing exacerbations of pulmonary sarcoidosis. Respirology 2008; 13 : 97-102.

35. Yeager H, Rossman MD, Baughman RP, et al. Pulmonary and psychosocial findings at enrollment in the ACCESS study. Sarcoidosis Vasc Diffuse Lung Dis 2005; 22: 147153.

36. Baughman RP, Sparkman BK, Lower EE. Six-minute walk test and health status assessment in sarcoidosis. Chest 2007; 132: 207-213.

37. Patil SN, Levin DL. Distribution of thoracic lymphadenopathy in sarcoidosis using computed tomography. J Thorac Imaging 1999; 14: 114-117. 